\title{
Bicuspid aortic valve and the specialty clinic: are your patients at risk?
}

\author{
Paul W. M. Fedak ${ }^{1,2}$ \\ ${ }^{1}$ Section of Cardiac Surgery, Department of Cardiac Sciences, Cumming School of Medicine, Libin Cardiovascular Institute \\ of Alberta, University of Calgary, Calgary, Alberta, Canada; ${ }^{2}$ Martha and Richard Melman Family Bicuspid Aortic \\ Valve Program, Blubm Cardiovascular Institute, Northwestern University, Chicago, Illinois, United States of America
}

Received: 3 January 2017; Accepted: 19 January 2017; First published online: 6 March 2017

W HEN SHOULD WE WORRY ABOUT THE AORTA IN patients with bicuspid aortic valve? Are there specific subgroups of patients at higher risk? When should we prophylactically replace the aorta in bicuspid aortic valve patients? These important questions are at the heart of most current research and discussion in the area of bicuspid aortic valve disease. As compared with the aorta, complications of the bicuspid aortic valve itself are more frequent and include valvular stenosis, regurgitation, or infection. Should patients and their caregivers be more concerned and vigilant about the valve or the associated aortopathy? What are the specific risks over time in a healthy asymptomatic ambulatory person in the community who is known to have a bicuspid aortic valve? Surprisingly, these questions are complex, and the answers remain somewhat elusive.

Rodriguez and co-workers examined the incidence and predictors of cardiac events using a retrospective analysis of ambulatory patients with bicuspid aortic valve followed-up at a specialty congenital clinic. Patients who underwent previous cardiac surgery either valve or aortic resection - were excluded. The primary aim of the study was to determine both the nature and the rate of disease progression in these selected patients. In addition, outcomes of specific interest in these patients were reported. Outcomes were defined as interventional - that is, intervention on the

Correspondence to: P. W. M. Fedak, MD, PhD, FRCSC, Section of Cardiac Surgery, Department of Cardiac Sciences, Cumming School of Medicine, Libin Cardiovascular Institute of Alberta, University of Calgary, C880, 1403-29 Street NW, Calgary, AB, Canada T2N 2T9. Tel: 403944 5931; Fax: 403270 3715; E-mail: paul.fedak@gmail.com aortic valve or the thoracic aorta - medical - such as death, aortic dissection, aortic valve endocarditis, congestive heart failure, arrhythmias, or ischaemic heart disease requiring hospital admission - and a composite end point of both. Over 200 patients were followed-up for more than 10 years. A multivariable analysis was used to identify independent predictors of the selected outcomes.

The main outcomes are reported in the manuscript. The clinical outcomes are not particularly surprising given the characteristics of the study cohort, but the specific risks are important and useful as a benchmark. The study has been expertly designed with an obvious recognition of the important aspects for bicuspid aortic valve patients. These data are useful for clinicians who have similar bicuspid aortic valve/congenital clinics. The outcomes are reported in a way that is patient-centric and may be of value to describe contemporary risks of key clinical end points to similar patients during consultations - for example, the understanding that $\sim 50 \%$ of such bicuspid aortic valve patients will have a medical or interventional event within 20 years is informative. This is what my patients want to know when I meet them in clinic, and with this contemporary study I can be more confident with my counsel.

There is currently controversy over the rate of disease progression in bicuspid aortic valve patients and actual risks of clinical events. Bicuspid aortic valve patients are known to be very heterogeneous, and surgical approaches can sometimes be at odds with established guidelines. ${ }^{1,2}$ The cohort of patients studied is critical to the analysis, and the reported outcomes and this may explain differences between 
published reports. In this study, at least half the patients were referred to the specialty clinic from a paediatric congenital clinic. Most others were referred because of valve disease/dysfunction. Only a small number of patients $(<10 \%)$ were referred to the clinic for known aortopathy, and $\sim 14 \%$ of patients had an ascending aorta size $>40 \mathrm{~mm}$ at baseline. Most of the patients were males and young. Many had an associated congenital lesion (30\%), but not complex lesions. This well-described cohort of bicuspid aortic valve patients is reflective of a community group found in an ambulatory specialty clinic setting.

Importantly, Michelena et al have observed different outcomes in bicuspid aortic valve patients depending on the stage and nature of their clinic referral. In their study, they show that outcomes are in fact different between a community cohort, a specialty clinic cohort, tertiary clinical referral, and a surgical-referral cohort of bicuspid aortic valve patients. ${ }^{3}$ Bicuspid aortic valve patients in the specialty clinic cohort, similar to the study by Rodriguez and colleagues, showed increased mortality as compared with the general population. This was not observed in the community cohort. The study by Rodriguez and colleagues helps inform us the actual risks for bicuspid aortic valve patients that are seemingly benign, but these patients are in fact a higher risk cohort found in our specially clinics.

Consistent with all retrospective clinical studies, this study has limitations that should be acknowledged. The study population was largely free of significant aortopathy at baseline. Not surprisingly, the data on outcomes show a predilection for valve events, and the multivariable analysis also favours valve progression. We must be cautious not to conclude that the associated aortopathy is benign and that the incidence of aortic progression is subclinical. Dilatation of the proximal aorta is present in $\sim 50 \%$ of individuals with a congenital bicuspid aortic valve. ${ }^{4}$ In some patients, severe aneurysmal aortic dilation may develop. Importantly, only one in every 1000 bicuspid aortic valve patients will experience aortic dissection per year. ${ }^{5}$ The overall incidence of aortic rupture/dissection is low among patients with bicuspid aortic valve, but the actual risk is increased as compared with patients with tricuspid aortic valves. Bicuspid aortic valve studies are routinely plagued by conclusions that do not appreciate the selection bias of the study population. For patients with minimal baseline aortopathy and some degree of valve dysfunction, these data are very appropriate. For patients with baseline aortopathy $(>45 \mathrm{~mm})$, these data may be misleading if applied as a predictive strategy.

In summary, Rodriguez and colleagues document long-term outcomes in bicuspid aortic valve patients from an ambulatory specialty clinic. They convincingly show that bicuspid aortic valve is not benign, and the majority of people with bicuspid aortic valve face a significant risk of clinical events that may require surgical interventions. The use of specialty bicuspid aortic valve clinics is warranted to provide routine surveillance and timely access to state-of-the-art care when appropriate.

\section{References}

1. Fedak PW. Bicuspid aortic valve syndrome: heterogeneous but predictable? Eur Heart J 2008; 29: 432-433.

2. Verma S, Yanagawa B, Kalra S, et al. Knowledge, attitudes, and practice patterns in surgical management of bicuspid aortopathy: a survey of 100 cardiac surgeons. J Thorac Cardiovasc Surg 2013; 146: 1033-1040.e4.

3. Michelena HI, Suri RM, Katan O, et al. Sex differences and survival in adults with bicuspid aortic valves: verification in 3 contemporary echocardiographic cohorts. J Am Heart Assoc 2016; 5: pii: e004211.

4. Verma S, Siu SC. Aortic dilatation in patients with bicuspid aortic valve. N Engl J Med 2014; 370: 1920-1929.

5. Michelena HI, Khanna AD, Mahoney D, et al. Incidence of aortic complications in patients with bicuspid aortic valves. JAMA 2011; 306: 1104-1112. 